while ii) poor baseline function as reflected by a higher health assessment questionnaire (HAQ) score was predictive of CVE with an AHR of 5.2 (95\% Cl 1.2-23). Conclusion: ERA patients treated by a T2T strategy did not develop excess CVE compared to CV risk factor-matched controls over 5 years. A longer disease remission duration was protective while a higher baseline HAQ was associated with a higher CVE risk.

REFERENCES:

[1] Agca $\mathrm{R}$ et al. EULAR recommendations for cardiovascular disease risk management in patients with rheumatoid arthritis and other forms of inflammatory joint disorders: 2015/2016 update. Annals of the Rheumatic Diseases.

Disclosure of Interests: None declared

DOI: 10.1136/annrheumdis-2021-eular.2040

\section{OP0104 DIFFERENCES IN LOW-DENSITY LIPOPROTEIN (LDL) PARTICLE COMPOSITION AND OXIDATION MAY UNDERLIE THE PARADOXICAL ASSOCIATION OF LOW LDL WITH HIGHER CORONARY ATHEROSCLEROSIS BURDEN IN RHEUMATOID ARTHRITIS}

G. Karpouzas ${ }^{1,2}$, S. Ormseth ${ }^{2}$, E. Hernandez ${ }^{2}$, M. Budoff ${ }^{3} .{ }^{1}$ Harbor-UCLA Medical Center, Rheumatology, Torrance, United States of America; ${ }^{2}$ The Lundquist Institute, Rheumatology, Torrance, United States of America; ${ }^{3}$ The Lundquist Institute, Cardiology, Torrance, United States of America

Background: The association between cholesterol and cardiovascular disease (CVD) risk is attenuated in Rheumatoid arthritis (RA). In fact, RA patients in the lowest low-density lipoprotein ( $\mathrm{LDL}$ ) group ( $<70 \mathrm{mg} / \mathrm{dl})$ may experience unexpectedly high CVD risk. Objectives: We here explored whether patients with $L D L<70 \mathrm{mg} / \mathrm{dl}$ (Group 1) had higher coronary atherosclerosis burden compared to other LDL groups (Group 2: $70 \leq L D L \leq 130$ and Group 3: $L D L>130$ ), as a reason for this risk. We further evaluated whether low LDL in group 1 associated with differences in inflammation, LDL particle composition or oxidation.

Methods: One hundred fifty RA patients without symptoms or history of CVD underwent coronary atherosclerosis evaluation with computed tomography angiography. Coronary artery calcium (CAC), number of segments with plaque (segment involvement score), stenotic severity (segment stenosis score), and extensive ( $>4$ segments with plaque) or obstructive disease ( $>50 \%$ stenosis) were assessed. Lipoprotein classes and subclasses were directly measured. Oxidized LDL (oxLDL) was measured with monoclonal antibody E06. Chemiluminescence Elisa quantified IgG and IgM antibodies to oxLDL (anti-oxLDL) and apoB100 immune complexes (IC). Proinflammatory cytokines were measured with Erenna Immunoassay. Robust linear and logistic regression modelsadjusted for Framingham D'Agostino score, obesity, disease activity, bDMARD and statin treatment- evaluated associations between LDL groups and plaque outcomes. Similar models evaluated adjusted differences in LDL subclasses, oxLDL, anti-oxLDL, anti-ApoB100 IC, and cytokines across LDL groups.

Results: Group 1 patients had higher coronary plaque burden (Figure $1 \mathrm{~A}$ ) and 2.8 times greater risk of extensive or obstructive disease (adjusted OR $2.82[95 \% \mathrm{Cl}$ 1.12-7.17], $P=0.031$ ) compared to LDL $>70$ groups. Among statin naïve patients,

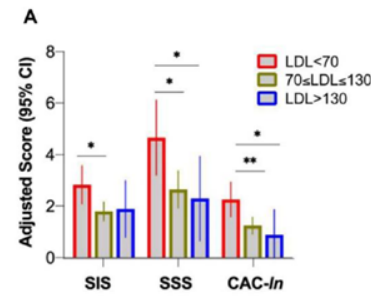

C

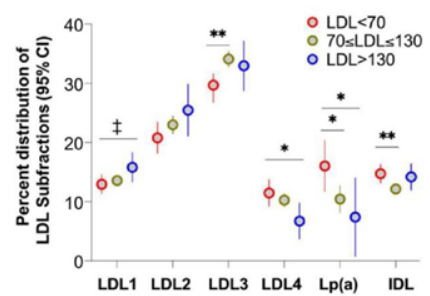

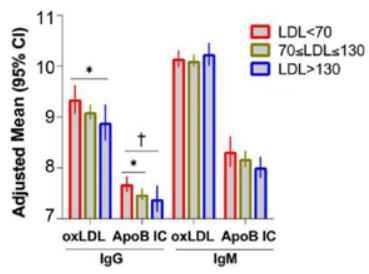

D LDL $<70$ vs. $70 \leq L D L \leq 130: p=0.005$
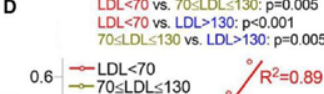

Figure 1 (A) Mean segment involvement score (SIS), segment stenosis score (SSS), and log transformed coronary artery calcium (CAC-In) across LDL groups, adjusted for Framingham-D'Agostino score, DAS28-CRP, waist-to-height ratio, bDMARD use, and statin use. (B) Mean serum levels of IgG and IgM anti-oxLDL and apoB100 immune complexes (IC) across LDL groups, adjusted for Framingham D'Agostino score and statin use. (C) LDL particle composition across LDL groups, adjusted for Framingham D'Agostino risk score, statin use, and total HDL-C. (D) Association between Lp(a) content and LDL oxidation across LDL groups, adjusted for Framingham D'Agostino score and statin use. $" p<0.050, "{ }^{*} p<0.010,{ }^{\dagger} p=0.051,{ }^{\ddagger} p=0.056$ those with LDL $<70$ also had higher oxLDL (log-transformed adjusted mean 2.55 [95\% Cl 2.34-2.77] versus 2.27 [95\% Cl 2.19-2.36], $P=0.018$ for LDL $>70$ ). Notably, Group 1 patients also had higher anti-oxLDL IgG and anti-ApoB100 IgG IC levels compared to other groups (Figure 1B). LDL subclass relative content in the LDL particle differed across groups (Figure 1C). Lp(a) was higher in LDL particles in Group 1 (adjusted mean 16.04\% [95\% Cl 11.75-20.33], versus 10.48\% [95\% $\mathrm{Cl} 8.20-12.75]$ in Group 2, $P=0.026$ and $7.41 \%$ [95\% Cl 0.77-14.04] in Group 3, $P=0.033)$. Notably, Lp(a) content strongly associated with oxLDL overall $(r=0.83, P<0.0001)$. This association was stronger for Group 1 compared to others $(P<0.005$, Figure 1D). No differences in RA activity, CRP, TNF- $\alpha$, IL-17A, or IL-17F were seen across groups. However, Group 1 had higher IL-6 (log-transformed adjusted mean $1.98[95 \% \mathrm{Cl}$ 1.64- 2.32] versus 1.57 [95\% $\mathrm{Cl} 1.45-1.70], P=0.028$ in Group 2 and 1.32 [95\% Cl $0.84-1.80], P=0.031$ in Group 3). IL-6 associated with both IgG anti-oxLDL ( $P=$ $0.015)$ and anti-apoB100 IC $(P=0.016)$. Log-transformed IL-6 further associated with higher log-transformed CAC (adjusted B 0.41 [95\% Cl 0.01-0.81], $P=0.049$ ). Conclusion: RA patients with $\mathrm{LDL}<70 \mathrm{mg} / \mathrm{dl}$ had higher coronary atherosclerosis burden. Low circulating LDL in that group may reflect higher oxidation; this was mostly linked to the larger $L p(a)$ relative content of LDL and its significantly higher oxidation potential in that group. OxLDL immune recognition was linked to higher IgG anti-oxLDL $A b$ and anti-ApoB100 IC levels in the $L D L<70$ group which further associated with higher IL-6 elaboration and atherosclerosis burden. Disclosure of Interests: George Karpouzas Speakers bureau: Sanofi/ Genzyme/ Regeneron, Consultant of: Sanofi/ Genzyme/ Regeneron, Grant/research support from: Pfizer, Sarah Ormseth: None declared, Elizabeth Hernandez: None declared, Matthew Budoff Consultant of: Pfizer DOI: 10.1136/annrheumdis-2021-eular.3397

\section{OP0105 FEASIBILITY AND USEFULNESS OF MAPPING BIOLOGIC REGISTRIES TO A COMMON DATA MODEL: ILLUSTRATION USING COMORBIDITIES}

L. Kearsley-Fleet ${ }^{1}$, K. Hyrich ${ }^{1,2}$, M. Schaefer ${ }^{3}$, D. Huschek ${ }^{3}$, A. Strangfeld ${ }^{3}$, J. Zavada ${ }^{4}$, M. Lagová ${ }^{5}$, D. Courvoisier ${ }^{6}$, C. Tellenbach ${ }^{7}$, K. Lauper ${ }^{1,6}$, C. SánchezPiedra $^{8}$, N. Montero ${ }^{8}$, J. T. Sánchez-Costa ${ }^{8}$, D. Prieto-Alhambra ${ }^{9,10}$, E. Burn ${ }^{10,11} .{ }^{1}$ The University of Manchester, Centre for Epidemiology Versus Arthritis, Manchester, United Kingdom; ${ }^{2}$ Manchester University NHS Foundation Trust, Manchester Academic Health Science Centre, Manchester, National Institute of Health Research Manchester Biomedical Research Centre, Manchester, United Kingdom; ${ }^{3}$ German Rheumatism Research Center (DRFZ Berlin), Epidemiology and Health Care Research Unit, Berlin, Germany; ${ }^{4}$ Institute of Rheumatology, Prague, Czech Republic; Institute of Biostatistics and Analyses Ltd, Brno, Czech Republic; ${ }^{6}$ University Hospitals of Geneva, Division of Rheumatology, Geneva, Switzerland; ${ }^{7}$ Swiss Clinical Quality Management in Rheumatic Diseases, Zurich, Switzerland; ${ }^{8}$ Spanish Society of Rheumatology, Madrid, Spain; ${ }^{9}$ Universitat Autonoma de Barcelona and Instituto de Salud Carlos III, GREMPAL Research Group, Idiap Jordi Gol and CIBERFes, Barcelona, Spain; ${ }^{10}$ University of Oxford, NDORMS, Oxford, United Kingdom; ${ }^{11}$ Fundació Institut Universitari per a la recerca a l'Atenció Primària de Salut Jordi Gol i Gurina, IDIAPJGol, Barcelona, Spain

Background: The Observational and Medical Outcomes Partnerships (OMOP) common data model (CDM) provides a framework for standardising health data with a view towards federated analyses, thus maximising the use and power of combining disparate datasets.

Objectives: To assess feasibility and usefulness of mapping biologic registry data from different European countries to the OMOP CDM and present initial descriptive data regarding comorbidities.

Methods: Five biologic registries, as part of a funded FOREUM project, have been mapped to the OMOP CDM: 1) the Czech biologics register (ATTRA), 2) Registro Español de Acontecimientos Adversos de Terapias Biológicas en Enfermedades Reumáticas (BIOBADASER), 3) British Society for Rheumatology Biologics Register for Rheumatoid Arthritis (BSRBR-RA), 4) German biologics register 'Rheumatoid arthritis observation of biologic therapy' (RABBIT), and 5) Swiss register 'Swiss Clinical Quality Management in Rheumatic Diseases' (SCQM). The mapping includes socio-demographic, observation period within the studies, baseline comorbidities, and baseline medications. Only patients with RA were included. Using $R$, registers received identical scripts to run on their mapped databases to produce an initial description of patient characteristics without the need to share patient-level data.

Results: A total of 54,458 individuals are included the five registries being mapped to the OMOP CDM, see table. Age and gender distribution was similar across registries. All registers reported on cardiovascular system comorbidities, diabetes mellitus, mental disorders, and respiratory system comorbidities. However, it was noted that results of comorbidity mapping relies on what each register collect on each patient at the point of registration.

Whilst the Charlson comorbidity index could be calculated within each registry, due to lack of the specific coding needed, such as "uncomplicated diabetes mellitus" / "end-organ damage diabetes mellitus", it was felt to be an inaccurate 